\title{
Nuclear Group Algebras for Finitely Generated Groups
}

\author{
Michel Cahen* \\ Département de Mathématique \\ Université Libre de Bruxelles \\ Campus Plaine, C. P. 218 \\ Boulevard du Triomphe \\ B-1050 Bruxelles \\ Belgium
}

\section{Simone Gutt ${ }^{\S}$}

\author{
Département de Mathématique \\ Université Libre de Bruxelles \\ Campus Plaine, C. P. 218 \\ Boulevard du Triomphe \\ B-1050 Bruxelles \\ Belgium
}

and

\author{
Stefan Waldmann \\ Institut für Mathematik \\ Universität Würzburg \\ Campus Hubland Nord \\ Emil-Fischer-Straße 31 \\ 97074 Würzburg \\ Germany
}

October 2016

\begin{abstract}
We study completions of the group algebra of a finitely generated group and relate nuclearity of such a completion to growth properties of the group. This extends previous work of Jolissaint on nuclearity of rapidly decreasing functions on a finitely generated group to more general weights than polynomial decrease. The new group algebras and their duals are studied in detail and compared to other approaches. As application we discuss the convergence of the complete growth function introduced by Grigorchuk and Nagnibeda.
\end{abstract}

\footnotetext{
${ }^{*}$ mcahen@ulb.ac.be

$\S$ sgutt@ulb.ac.be

TStefan. Waldmann@mathematik . uni-wuerzburg. de
} 


\section{Contents}

1 Introduction

2 Notation and Preliminaries

3 Growth Functions

4 The General Construction

5 Comparison with Previous Results 11

6 Properties of $\mathcal{A}_{\sigma} \quad$

$\begin{array}{lll}7 & \text { The Dual } & 16\end{array}$

8 The Spaces $\mathfrak{c}_{L, \sigma,-R}^{0}(G) \quad \square$

9 An Application: The Complete Growth

A Some Locally Convex Analysis $\quad 22$

\begin{tabular}{ll} 
References & 23 \\
\hline
\end{tabular} 


\section{Introduction}

In [6, 7] Jolissaint introduced and studied a completion of the group algebra of a finitely generated group resembling the construction of the Schwartz space of rapidly decreasing functions: the coefficients decay faster than every polynomial in the length of the group element, where one has to choose a length functional for the group, e.g. based on the choice of finitely many generators. This algebra turned out to be an extremely useful tool to investigate other completions of the group algebra like the (reduced) $C^{*}$-group algebra. Among many other results Jolissaint showed that the Fréchet space of such rapidly decreasing functions is nuclear iff the group has polynomial growth.

In this paper we generalize Jolissaint's constructions and results by taking into account more general types of decays for the coefficients in the group algebra.

To this end we consider growth functions $\sigma$ on the integers which are either sub-multiplicative or almost sub-multiplicative (in the sense of (3.6)). In both cases we define, for a given choice of a length functional $L$ on the group and a parameter $R \geq 0$, a weighted $\ell^{1}$-norm

$$
\|a\|_{L, \sigma, R}=\sum_{g \in G}\left|a_{g}\right| \sigma(L(g))^{R}
$$

for $a \in \mathbb{C}[G]$. The system of all such norms for $R \in[0, \infty)$ yield an algebra topology on the group algebra $\mathbb{C}[G]$ which turns out to be independent of $L$. The completion is shown to be a Fréchet-Hopf *-algebra $\mathcal{A}_{\sigma}(G)$. We characterize the nuclearity of the completion $\mathcal{A}_{\sigma}(G)$ by comparing the growth of $\sigma$ to the growth of the group: we have nuclearity iff $\sigma$ grows at least as fast as the group (in the sense of (3.8)). The basic idea of the proof is to realize the algebra $\mathcal{A}_{\sigma}(G)$ as a certain Köthe space such that one can apply the easy Grothendieck-Pietsch criterion for nuclearity. We also characterize the dual and its rich bimodule structure quite explicitly and give as a first application convergence results for the complete growth function, introduced by Grigorchuk and Nagnibeda in [4], as an element of the dual: again, we get convergence and thus a holomorphic complete growth function iff the growth of $\sigma$ is at least as fast as the one of the group.

The paper is organized as follows: in Section 2 we explain our notation and recall some basic facts on finitely generated groups to set-up the stage. In section 3 we specify the kind of growth functions which we are going to use and discuss many examples. The general construction of the norms and completions of the group algebra is presented in Section 4 . In Section 5 we compare our construction to yet another version from [1]; understanding the construction presented there was one of the motivations to start the present work. Section [ contains the main result on the nuclearity as well as a discussion of the canonical Schauder basis of $\mathcal{A}_{\sigma}(G)$. The dual and its inductive limit topology are described in Section 7 . Section 8 contains a detailed analysis of sub-bimodules of the dual which can be seen as analogs of spaces of continuous functions vanishing (in a weighted sense) at infinity. Finally, we prove the convergence statements for the complete growth function in Section 9 , In a small appendix we collect some background material in locally convex analysis of sequence spaces. Acknowledgements: This research was partially supported by the IAP programme "Dygest" of the Belgium Science Policy Office.

\section{Notation and Preliminaries}

In this section we will recall some basic notions from the theory of discrete groups and their group algebras. Let $G$ be a discrete group, i.e. we consider the group as a discrete topological space and hence as a topological group. Later on, we will be interested in finitely generated groups, but for the time being, this is not yet important. We consider the group algebra $\mathbb{C}[G]$ defined to be the complex vector space spanned by the set $G$ and endowed with the multiplication inherited from $G$. More precisely, we have the basis vectors $\mathrm{e}_{g} \in \mathbb{C}[G]$, where $g \in G$, and the product of $a, b \in \mathbb{C}[G]$ is given 
by

$$
a b=\left(\sum_{g \in G} a_{g} \mathrm{e}_{g}\right)\left(\sum_{h \in G} b_{h} \mathrm{e}_{h}\right)=\sum_{g, h \in G} a_{g} b_{h} \mathrm{e}_{g h}=\sum_{h \in G}\left(\sum_{g \in G} a_{g} b_{g^{-1} h}\right) \mathrm{e}_{h},
$$

where only finitely many of the coefficients $a_{g}$ and $b_{h}$ are different from zero. We denote the coefficient functionals by $\delta_{g}: \mathbb{C}[G] \ni a \mapsto a_{g} \in \mathbb{C}$.

The product (2.1) makes the group algebra $\mathbb{C}[G]$ a unital associative algebra where the unit element is given by the group unit, i.e. by $\mathrm{e}_{e}$. Moreover, $\mathbb{C}[G]$ becomes a cocommutative Hopf *algebra by the following structure maps: the ${ }^{*}$-involution is given by $a^{*}=\sum_{g \in G} \overline{a_{g}} \mathrm{e}_{g^{-1}}$, the antipode is $S(a)=\sum_{g \in G} a_{g} \mathrm{e}_{g^{-1}}$, the counit is $\epsilon(a)=\sum_{g \in G} a_{g}$, and the coproduct is $\Delta(a)=\sum_{g \in G} a_{g} \mathrm{e}_{g} \otimes \mathrm{e}_{g}$. The group-like elements of $\mathbb{C}[G]$ with respect to this coproduct are then precisely the group elements, i.e. the basis vectors $\mathrm{e}_{g}$ for $g \in G$. Note that the tensor product $\mathbb{C}[G] \otimes \mathbb{C}[G]$ is canonically isomorphic as algebra to $\mathbb{C}[G \times G]$ where $G \times G$ is endowed with the product group structure.

In the following it will be advantageous to consider also the formal power series in $G$ which we denote by $\mathbb{C}[[G]]$ : this is the vector space of formal series $a=\sum_{g \in G} a_{g} \mathrm{e}_{g}$ where now $a_{g}$ is unrestricted. Then $\mathbb{C}[[G]]$ is no longer an algebra but the multiplication (2.1) extends to a bimodule structure for $\mathbb{C}[[G]]$ over $\mathbb{C}[G]$.

The formal power series $\mathbb{C}[[G]]$ can also be identified with the algebraic dual of $\mathbb{C}[G]$ as follows. A linear functional $\Phi: \mathbb{C}[G] \longrightarrow \mathbb{C}$ is determined by its values $\varphi_{g}=\Phi\left(\mathrm{e}_{g}\right)$ on the basis $\left\{\mathrm{e}_{g}\right\}_{g \in G}$. This defines a formal series $\varphi=\sum_{g \in G} \varphi_{g} \mathrm{e}_{g}$ such that for all $a \in \mathbb{C}[G]$ one has

$$
\Phi(a)=\sum_{g \in G} a_{g} \Phi\left(\mathrm{e}_{g}\right)=\sum_{g \in G} a_{g} \varphi_{g}=\langle\varphi, a\rangle
$$

where we take the last equation as definition for the bilinear pairing $\langle\cdot, \cdot\rangle: \mathbb{C}[[G]] \times \mathbb{C}[G] \longrightarrow \mathbb{C}$. Conversely, every $\varphi \in \mathbb{C}[[G]]$ gives a linear functional $\Phi$ on $\mathbb{C}[G]$ by this equation. Using the coefficient functionals, the linear functional $\Phi$ corresponding to $\varphi$ is just $\Phi=\sum_{g \in G} \varphi_{g} \delta_{g}$. The infinite series becomes finite after application to an element in $\mathbb{C}[G]$.

While this is true for every vector space with basis, we have now the additional bimodule structures of $\mathbb{C}[[G]]$ and of the dual: clearly the dual $\mathcal{A}^{*}$ of an algebra $\mathcal{A}$ is always a bimodule over the algebra by

$$
(a \triangleright \Phi \triangleleft b)(c)=\Phi(b c a)
$$

for $a, b, c \in \mathcal{A}$ and $\Phi \in \mathcal{A}^{*}$. We can relate this canonical bimodule structure to the one of $\mathbb{C}[[G]]$ as follows. For later use we introduce already here the trace functional

$$
\operatorname{tr}: \mathbb{C}[[G]] \ni a \mapsto a_{e} \in \mathbb{C} .
$$

With some abuse of notation we have $\operatorname{tr}=\delta_{e}$, but note that $\delta_{e}$ is a linear functional on $\mathbb{C}[G]$ while $\operatorname{tr}$ is defined on $\mathbb{C}[[G]]$. It is now easy to see that $\operatorname{tr}$ is indeed a trace in the sense that for $a \in \mathbb{C}[G]$ and $b \in \mathbb{C}[[G]]$ we have

$$
\operatorname{tr}(a b)=\operatorname{tr}(b a)
$$

where we use the above bimodule structure of $\mathbb{C}[[G]]$. In particular, tr restricts to a trace on the algebra $\mathbb{C}[G]$ given by $\left.\operatorname{tr}\right|_{\mathbb{C}[G]}=\delta_{e}$. For the bimodule structure we note that

$$
\mathbb{C}[[G]] \ni b \mapsto\langle S(b), \cdot\rangle=\operatorname{tr}(b \cdot) \in(\mathbb{C}[G])^{*}
$$

is now an isomorphism of bimodules, where we use the canonical bimodule structures for both sides. Note that the use of the antipode is crucial here. As a consequence, we can embed the group algebra $\mathbb{C}[G]$ into its algebraic dual $(\mathbb{C}[G])^{*}$ as a bimodule. 
In the following we want to study completions of $\mathbb{C}[G]$ with respect to various topologies. The easiest case is well-known and given by the $\ell^{1}$-like group algebra: we want to extend the counit $\epsilon$ to an as large as possible subspace of $\mathbb{C}[[G]]$ such that the formula $\epsilon(a)=\sum_{g \in G} a_{g}$ still makes sense. Since we do not require a specific order of summation, we have either unconditional convergence or divergence: but in $\mathbb{C}$ unconditional convergence is the same as absolute convergence. Moreover, we know that at most countably many contributions can be different from zero. This motivates the following definition: for $a \in \mathbb{C}[[G]]$ one defines

$$
\|a\|_{\ell^{1}(G)}=\sum_{g \in G}\left|a_{g}\right| \in[0,+\infty]
$$

and sets $\ell^{1}(G)=\left\{a \in \mathbb{C}[[G]] \mid\|a\|_{\ell^{1}(G)}<\infty\right\}$. Then it is easy to see that $\|\cdot\|_{\ell^{1}(G)}$ is a norm on $\ell^{1}(G)$ turning it into a Banach space with $\mathbb{C}[G]$ being a dense subspace. Moreover, $|\epsilon(a)| \leq\|a\|_{\ell^{1}(G)}$ shows that $\epsilon$ is a continuous linear functional on $\ell^{1}(G)$. The space $\ell^{1}(G)$ is optimal with respect to this property: it is the largest subspace to which $\epsilon$ can be extended by means of the convergent series. The basis vectors $\mathrm{e}_{g}$ of $\mathbb{C}[G]$ constitute an absolute Schauder basis of $\ell^{1}(G)$, i.e. the coefficient functionals $\delta_{g}$ are continuous and $a=\sum_{g \in G} \delta_{g}(a) \mathrm{e}_{g}$ converges absolutely for all $a \in \ell^{1}(G)$ such that $\sum_{g \in G}\left|\delta_{g}(a)\right|\left\|\mathrm{e}_{g}\right\|_{\ell^{1}(G)}$ can be estimated by the norm: it simply coincides with $\|a\|_{\ell^{1}(G)}$. Note that for $a \in \ell^{1}(G)$ at most countably many coefficients $\delta_{g}(a)=a_{g}$ are different from zero.

The remaining algebraic features of $\mathbb{C}[G]$ extend continuously to $\ell^{1}(G)$ : the product is continuous making $\ell^{1}(G)$ a Banach algebra, the *-involution as well as the antipode are continuous with operator norm 1 , and the coproduct extends to $\ell^{1}(G)$ taking now values in $\ell^{1}(G) \hat{\otimes}_{\pi} \ell^{1}(G)=\ell^{1}(G \times G)$ : here we have to use the completed projective tensor product of the Banach space $\ell^{1}(G)$ with itself and we identified the $\ell^{1}$-norm on $\mathbb{C}[G \times G]$ with the tensor products of the $\ell^{1}$-norms according to Lemma A.1. Then we have the equality $\|\Delta(a)\|_{\ell^{1}(G \times G)}=\|a\|_{\ell^{1}(G)}$ for all $a \in \ell^{1}(G)$, which proves the continuity of $\Delta$. This way we obtain a Banach-Hopf ${ }^{*}$-algebra $\ell^{1}(G)$. It is now fairly easy to see that also in this completion the group-like elements are simply given by the basis vectors $\left\{\mathrm{e}_{g}\right\}_{g \in G}$ as before. Thus also $\ell^{1}(G)$ allows to recover $G$ as a group.

\section{Growth Functions}

In this section we recall the basic properties of the word length in a finitely generated group and the resulting surface and volume growth properties, see e.g. [2, Chap. VI]. Moreover, we will give a class of growth functions to which we want to compare the growth properties of a finitely generated group.

Let $G$ be a finitely generated group with a finite set $S$ of generators. The choice of $S$ then gives a word length $L: G \longrightarrow \mathbb{N}_{0}$ by taking the minimal number $L(g)$ of generators needed to write $g \in G$ as a product of generators. By convention, $L(e)=0$ for the group unit $e \in G$ and we assume that $S=S^{-1}$. Then $L$ enjoys the properties

$$
L(g)=L\left(g^{-1}\right) \quad \text { and } \quad L(g h) \leq L(g)+L(h)
$$

for all $g, h \in G$. These properties allow to define a word metric $d: G \times G \longrightarrow \mathbb{N}_{0}$ by setting

$$
d(g, h)=L\left(g^{-1} h\right)
$$

for $g, h \in G$. If $L^{\prime}$ is another word length functional corresponding to a different set of generators $S^{\prime}$ then we have constants $c, c^{\prime} \in \mathbb{N}$ with $L(g) \leq c L^{\prime}(g)$ and $L^{\prime}(g) \leq c^{\prime} L(g)$ for all $g \in G$. This implies that the two word metrics give the same coarse geometry: we have estimates $d(g, h) \leq c d^{\prime}(g, h)$ and $d^{\prime}(g, h) \leq c^{\prime} d(g, h)$ for all $g, h \in G$.

Using the word length one defines the surface growth and the volume growth of the group $G$ by

$$
\sigma_{G}(n)=\#\{g \in G \mid L(g)=n\} \quad \text { and } \quad \beta_{G}(n)=\#\{g \in G \mid L(g) \leq n\} \text {, }
$$


where we suppress the dependence on $L$ in the notation. We have $\sigma_{G}(n) \leq \beta_{G}(n)$ and $\beta_{G}(n)$ grows at most exponentially in $n$. Since we assume $G$ to be infinite, we have $\sigma_{G}(n) \geq 1$ for all $n$ and $\beta_{G}$ is strictly increasing. In particular $\beta_{G}(n)>n$. Moreover, we have the following crucial properties

$$
\sigma_{G}(n+m) \leq \sigma_{G}(n) \sigma_{G}(m) \quad \text { and } \quad \beta_{G}(n+m) \leq \beta_{G}(n) \beta_{G}(m)
$$

for all $n, m \in \mathbb{N}_{0}$.

We shall now generalize the properties of the functions $\sigma_{G}$ and $\beta_{G}$ in order to find a class of functions to which we can compare them. Here we will not use the standard way of comparing growth functions, as this is usually done in geometric group theory, see [2, Chap. VI]. Instead, we will need a slightly coarser notion.

There are two aspects of importance here: the surface growth as well as the volume growth are submultiplicative. The volume growth is also monotonic. In general, we call a map $\sigma: \mathbb{N}_{0} \longrightarrow[0, \infty)$ submultiplicative if

$$
\sigma(n+m) \leq \sigma(n) \sigma(m)
$$

for all $n, m \in \mathbb{N}_{0}$ and almost submultiplicative if for every $\epsilon>0$ there is a constant $c>0$ such that

$$
\sigma(n+m) \leq c \sigma(n)^{1+\epsilon} \sigma(m)^{1+\epsilon}
$$

for all $n, m \geq \mathbb{N}_{0}$. By induction, we conclude that for an almost submultiplicative map $\sigma$ we get for every $\epsilon>0$ and every $m \in \mathbb{N}$ a constant $c_{\epsilon, m}>0$ with

$$
\sigma(n m) \leq c_{\epsilon, m} \sigma(n)^{m+\epsilon}
$$

for all $n \geq 0$. The almost submultiplicative functions will allow for a slightly greater flexibility later on. Clearly, a submultiplicative function is also almost submultiplicative. It will be important to consider only functions $\sigma$ which are non-zero everywhere. Moreover, we will need that $\sigma$ is monotonic: here we use monotonic in the sense of $\sigma(n) \leq \sigma(n+1)$ and emphasize the strict monotonic case $\sigma(n)<\sigma(n+1)$ whenever it is actually needed.

Definition 3.1 (Growth function) A map $\sigma: \mathbb{N}_{0} \longrightarrow[1, \infty)$ is called a growth function if it is monotonically increasing and unbounded.

Later on, we will add some additional conditions concerning the speed how fast $\sigma$ diverges to $+\infty$ for large arguments.

Example 3.2 (Growth functions) The following examples of growth functions will be of particular importance in this work:

i.) The volume growth $\beta_{G}$ of a finitely generated infinite group is a growth function which increases even strictly monotonically. Moreover, this growth function is submultiplicative. While the surface growth $\sigma_{G}$ is submultiplicative, it will not be a growth function in general, since there is no need for $\sigma_{G}$ to be unbounded: e.g. for $G=(\mathbb{Z},+)$ the surface growth with respect to the standard generators $S=\{1,-1\}$ is constant $\sigma_{\mathbb{Z}}(n)=2$.

ii.) If $\sigma$ and $\tau$ are growth functions, then $x \sigma+y \tau$ is a growth function for all $x, y \geq 1$. Moreover, $\sigma \tau$ and $\sigma^{d}$ for $d>0$ are growth functions.

iii.) The map $\sigma(n)=1+n$ is a submultiplicative growth function, called the linear growth function. Note that we use $1+n$ in order to have non-zero values everywhere. Also $\sigma(n)=a_{d} n^{d}+\cdots+$ $a_{1} n+1$ is a growth function whenever $a_{d}, \ldots, a_{1} \geq 0$ and $a_{d}>0$, which we call a polynomial growth of degree $d$.

iv.) Let $0<\theta \leq 1$ then $\sigma(n)=\exp \left(n^{\theta}\right)$ is a submultiplicative growth function. This is called the exponential growth for $\theta=1$ and a sub-exponential growth for $\theta<1$. 
v.) The factorial $\sigma(n)=n$ ! is a growth function, called the factorial growth. In fact, it is not submultiplicative but almost submultiplicative: this follows since a binomial coefficient can always be dominated by an arbitrarily small power of a factorial. In fact, for all $\epsilon>0$ we have $(n+m) ! \leq c_{\epsilon}(n !)^{1+\epsilon}(m !)^{1+\epsilon}$ where $c_{\epsilon}$ is chosen by e.g. $c_{\epsilon}=\sup _{n, m} \frac{2^{n+m}}{n !^{\epsilon} m !^{\epsilon}}<\infty$.

vi.) With the convention $x !=\Gamma(x+1)$ for $x \in \mathbb{R}$, we can obtain a growth function for every $0<\theta \leq 1$ by setting $\sigma(n)=\left(n^{\theta}\right)$ !. Since the $\Gamma$-function is (strictly) monotonically increasing for arguments $x \geq 2$, we obtain a strictly increasing growth function. We call these growth functions sub-factorial.

vii.) If $\tau: \mathbb{N}_{0} \longrightarrow[0, \infty)$ is subadditive, i.e. $\tau(n+m) \leq \tau(n)+\tau(m)$ and if $\sigma:[0, \infty) \longrightarrow[0, \infty)$ is submultiplicative then $\sigma \circ \tau$ is submultiplicative again. If $\sigma$ was only almost submultiplicative, then $\sigma \circ \tau$ is almost submultiplicative again. It follows that $\sigma$ from the previous part is an almost submultiplicative growth function for all $0<\theta \leq 1$. This can of course be checked directly as well. In fact, these (sub-) factorial growth functions constitute one of our main motivations.

We need to establish a reasonable notion of equivalence which measures the type of growth of $\sigma$. Thus let $\sigma, \sigma^{\prime}: \mathbb{N}_{0} \longrightarrow[1, \infty)$ be two maps. We define now a relation $\preccurlyeq$ by $\sigma \preccurlyeq \sigma^{\prime}$ if there are constants $c, k \geq 1$ with

$$
\sigma(n) \leq c \sigma^{\prime}(c n)^{k}
$$

for all $n \in \mathbb{N}_{0}$. Though we are mainly interested in growth functions, we will use this relation also for non-monotonic functions.

It is now easy to see that this gives a transitive and reflexive relation. We will call two functions $\sigma$ and $\sigma^{\prime}$ equivalent if we have $\sigma \preccurlyeq \sigma^{\prime}$ and $\sigma^{\prime} \preccurlyeq \sigma$. In this case we write $\sigma \sim \sigma^{\prime}$. Note that our notion of equivalence and $\preccurlyeq$ is slightly coarser than the usual notion employed in geometric group theory, see [2, Chap. VI]. In fact, we get back to the more common notion if we allow only for $k=1$ in (3.8). However, it will be important to work with this less restrictive notion to compare growth functions. In particular, we always have

$$
\sigma \sim \sigma^{d}
$$

for all $d \geq 1$.

It is now easy to see that the relation $\preccurlyeq$ is compatible with pointwise convex combinations, products, and powers, i.e. we have for $\sigma \preccurlyeq \tau$ and $\sigma^{\prime} \preccurlyeq \tau^{\prime}$ and $a, b, d \geq 1$ also

$$
\sigma \sigma^{\prime} \preccurlyeq \tau \tau^{\prime}, \quad a \sigma+b \sigma^{\prime} \preccurlyeq a \tau+b \tau^{\prime}, \quad \text { and } \quad \sigma^{d} \preccurlyeq \tau^{d} .
$$

Quite different from the usual notion for comparing growth functions, the relation $\preccurlyeq$ is in-sensitive to different polynomial growths:

Lemma 3.3 Let $\sigma$ be a growth function which grows at least as $(1+n)^{\theta}$ for some $\theta>0$, i.e. for which we have $(1+n)^{\theta} \preccurlyeq \sigma$. Then for all polynomial growth functions $p$ we have $p \sigma \sim \sigma$. In particular, all polynomial growth functions are equivalent.

PROOF: This is just a repeated application of (3.10).

On the other hand, the equivalence relation $\sim$ is sensitive to different types of super-polynomial growths. In particular, for all $0<\theta<\theta^{\prime}$ we have for every polynomial growth $p$

$$
1 \preccurlyeq p \preccurlyeq \exp \left(n^{\theta}\right) \preccurlyeq \exp \left(n^{\theta^{\prime}}\right),
$$

without having equivalence. Moreover, in between the different types of sub-exponential growths we have the sub-factorial ones

$$
\exp \left(n^{\theta}\right) \preccurlyeq\left(n^{\theta}\right) ! \preccurlyeq \exp \left(n^{\theta^{\prime}}\right)
$$


for $0<\theta<\theta^{\prime}$, again without having equivalence. This gives us a wealth of inequivalent growth functions, both for the submultiplicative and the almost submultiplicative case.

Finally, we recall that a submultiplicative map grows at most exponentially, i.e. we have $\sigma(n) \leq c^{n}$ with $c=\max \{\sigma(0), \sigma(1)\}$. An almost submultiplicative map can grow faster than exponentially like e.g. $\sigma(n)=n$ !. However, also the growth of an almost submultiplicative map is bounded e.g. by $\sigma(n) \leq c^{2^{n}}$ for some suitable $c>0$.

\section{The General Construction}

In this section we present the main construction of various other group algebras for $G$ based on the following simple idea: the $\ell^{1}$-completion is the largest possible completion to have the counit well-defined by means of the series expression. Hence we are looking now for finer topologies than the $\ell^{1}$-topology which will still give us the structure of locally convex algebras and, hopefully, Hopf algebras. The topologies will be determined by means of seminorms which are analogs of the $\ell^{1}$-norm but involve now different weights for each basis vector $\mathrm{e}_{g}$ instead of the uniform counting measure.

The main idea is to find weights depending on the word length of the group element in such a way that we get seminorms allowing for a continuous multiplication. To guarantee a continuous product we use a submultiplicative or an almost submultiplicative growth function $\sigma$. We fix a set of generators with corresponding word length $L$ on $G$. Moreover, we consider $R \geq 0$ and set

$$
\|a\|_{L, \sigma, R}=\sum_{g \in G}\left|a_{g}\right| \sigma(L(g))^{R}
$$

for $a \in \mathbb{C}[[G]]$, allowing for the value $+\infty$. We set

$$
\ell_{L, \sigma, R}^{1}(G)=\left\{a \in \mathbb{C}[[G]] \mid\|a\|_{L, \sigma, R}<\infty\right\} .
$$

Then clearly $\ell_{L, \sigma, R}^{1}(G)$ is a subspace of $\mathbb{C}[[G]]$ containing $\mathbb{C}[G]$ and $\|\cdot\|_{L, \sigma, R}$ is a norm on it, turning $\ell_{L, \sigma, R}^{1}(G)$ into a Banach space with $\mathbb{C}[G]$ being a dense subspace. Moreover,

$$
\|a\|_{\ell^{1}(G)} \leq\|a\|_{L, \sigma, R}
$$

for all $a \in \mathbb{C}[[G]]$ and hence $\ell_{L, \sigma, R}^{1}(G) \subseteq \ell^{1}(G)$ is a continuous inclusion. Note that the property $\sigma \geq 1$ for a growth function becomes crucial here. For $R=0$ we have equality of the norms and hence of the Banach spaces. Similarly, for $R \geq R^{\prime}$ we have

$$
\|a\|_{L, \sigma, R^{\prime}} \leq\|a\|_{L, \sigma, R}
$$

leading to the continuous inclusion $\ell_{L, \sigma, R}^{1}(G) \subseteq \ell_{L, \sigma, R^{\prime}}^{1}(G)$. Finally, it is clear that the ${ }^{*}$-involution, the counit, and the antipode are continuous with respect to the norms $\|\cdot\|_{L, \sigma, R}$ for all choices of $\sigma$ and $R$ as above. In fact, $\left\|a^{*}\right\|_{L, \sigma, R}=\|a\|_{L, \sigma, R}=\|S(a)\|_{L, \sigma, R}$ since we have $L\left(g^{-1}\right)=L(g)$. The continuity of the product is slightly more tricky: here we need to use the (almost) submultiplicativity of $\sigma$ :

Lemma 4.1 Let $\sigma$ be a growth function and let $R \geq 0$.

i.) If $\sigma$ is submultiplicative then $\|a b\|_{R, \sigma, L} \leq\|a\|_{L, \sigma, R}\|b\|_{L, \sigma, R}$ for all $a, b \in \mathbb{C}[[G]]$ and hence $\ell_{L, \sigma, R}^{1}(G)$ is a Banach *-algebra.

ii.) If $\sigma$ is almost submultiplicative then for every $\epsilon>0$ there is a constant $c>0$ such that $\|a b\|_{R, \sigma, L} \leq c\|a\|_{L, \sigma, R+\epsilon}\|b\|_{L, \sigma, R+\epsilon}$ for all $a, b \in \mathbb{C}[[G]]$. 
Proof: The proof consist in a straightforward estimate using $\sigma(L(h)) \leq \sigma\left(L(g)+L\left(g^{-1} h\right)\right)$ and the (almost) submultiplicativity of $\sigma$.

It follows that in the almost submultiplicative case we do not get a normed algebra. However, there is a rather easy way out: we have to pass to a suitable projective limit which is possible thanks to the continuous inclusions $\ell_{L, \sigma, R}^{1}(G) \subseteq \ell_{L, \sigma, R^{\prime}}^{1}(G)$ for $R \geq R^{\prime}$. Hence, for a fixed $R>0$, we take the intersection

$$
\ell_{L, \sigma, R^{-}}^{1}(G)=\bigcap_{R \geq \epsilon>0} \ell_{L, \sigma, R-\epsilon}^{1}(G)
$$

and equip it with the projective limit topology: more explicitly, this is determined by all the norms $\|\cdot\|_{L, \sigma, R-\epsilon}$ for $0<\epsilon \leq R$. Since then for all $\delta>0$ we find an $0<\epsilon<\delta$ and a constant $c>0$ with

$$
\|a b\|_{L, \sigma, R-\delta} \leq c\|a\|_{L, \sigma, R-\epsilon}\|b\|_{L, \sigma, R-\epsilon}
$$

we conclude that the product is continuous in the projective limit topology. Moreover, the estimate from (4.4) shows that countably many of the seminorms are sufficient to determine the topology:

Corollary 4.2 For an almost submultiplicative growth function $\sigma$ and $R>0$ the space $\ell_{L, \sigma, R^{-}}^{1}(G)$ is a unital Fréchet ${ }^{*}$-algebra with $\mathbb{C}[G]$ being a dense subalgebra. We have continuous inclusions $\ell_{L, \sigma, R^{-}}^{1}(G) \subseteq \ell_{L, \sigma, R^{\prime}}^{1}(G)$ whenever $R \geq R^{\prime}$. The counit $\epsilon$ and the antipode $S$ are continuous.

We come now to the question whether the coproduct is still continuous to make these new algebras also Banach-Hopf or Fréchet-Hopf algebras. Here things turn out to be slightly more complicated. As before we use the tensor product of the norm $\|\cdot\|_{L, \sigma, R}$ with itself to get a norm on $\mathbb{C}[G \times G]$. From Lemma A.1 we then get

$$
\|\Delta(a)\|_{L, \sigma, R}=\sum_{g, h \in G}\left|\Delta(a)_{g, h}\right| \sigma(L(g))^{R} \sigma(L(h))^{R}=\sum_{g \in G}\left|a_{g}\right| \sigma(L(g))^{2 R}=\|a\|_{L, \sigma, 2 R}
$$

for all $a \in \mathbb{C}[G]$. Thus the coproduct extends to a continuous linear map

$$
\Delta: \ell_{L, \sigma, R}^{1}(G) \longrightarrow \ell_{L, \sigma, 2 R}^{1}(G) \hat{\otimes}_{\pi} \ell_{L, \sigma, 2 R}^{1}(G),
$$

but in general it will not be continuous for the (strictly) finer topology determined by the norm $\|\cdot\|_{L, \sigma, R}$ on the target side. Hence $\ell_{L, \sigma, R}^{1}(G)$ will not be a Banach-Hopf algebra unless $R=0$.

Nevertheless, the estimate (equality) in (4.8) gives a hint how to establish the continuity: we have to take again a projective limit, now $R \longrightarrow \infty$, in order to have all seminorms $\|\cdot\|_{L, \sigma, R}$ at our disposal.

Definition 4.3 (The algebra $\mathcal{A}_{\sigma}$ ) Let $\sigma$ be an almost submultiplicative growth function. Then we define

$$
\mathcal{A}_{\sigma}(G)=\left\{a \in \mathbb{C}[[G]] \mid\|a\|_{L, \sigma, R}<\infty \text { for all } R \geq 0\right\}=\bigcap_{R \geq 0} \ell_{L, \sigma, R}^{1}(G),
$$

equipped with the projective locally convex topology of all the seminorms $\|\cdot\|_{L, \sigma, R}$.

We will now investigate some first properties of $\mathcal{A}_{\sigma}(G)$. The most important is that even though the individual spaces $\ell_{L, \sigma, R}^{1}(G)$ depend on the choice of the set of generators and hence on $L$, this dependence disappears for $\mathcal{A}_{\sigma}$ : suppose that $L^{\prime}$ is another word length obtained from a different set of generators. Then we have $\sigma(m n) \leq \sigma(n)^{m}$ for all $n \in \mathbb{N}_{0}$ in the case $\sigma$ is submultiplicative. For an almost submultiplicative $\sigma$ we get for all $\epsilon>0$ a constant $c>0$ depending on $m$ with $\sigma(m n) \leq c \sigma(n)^{m+\epsilon}$ for all $n \in \mathbb{N}_{0}$. In the submultiplicative case this gives

$$
\|a\|_{L, \sigma, R}=\sum_{g \in G}\left|a_{g}\right| \sigma(L(g))^{R} \leq \sum_{g \in G}\left|a_{g}\right| \sigma\left(c L^{\prime}(g)\right)^{R} \leq \sum_{g \in G}\left|a_{g}\right| \sigma\left(L^{\prime}(g)\right)^{c R}=\|a\|_{L^{\prime}, \sigma, c R},
$$


where we used the monotonicity of $\sigma$ and the constant $c$ from $L(g) \leq c L^{\prime}(g)$. Using (3.7), in the almost submultiplicative case we get for all $\epsilon>0$ another constant $c^{\prime}>0$ with

$$
\|a\|_{L, \sigma, R} \leq\|a\|_{L^{\prime}, \sigma, c^{\prime} R+\epsilon} .
$$

In both cases we can get the following statement for the projective limit $R \longrightarrow \infty$ :

Proposition 4.4 Let $\sigma$ be an almost submultiplicative growth function.

i.) The projective limit $\mathcal{A}_{\sigma}(G)$ of the Banach spaces $\ell_{L, \sigma, R}^{1}(G)$ is independent of the chosen word length $L$.

ii.) The group algebra $\mathbb{C}[G]$ is dense in $\mathcal{A}_{\sigma}(G)$ and all algebraic structures are continuous yielding a Fréchet-Hopf *-algebra structure on $\mathcal{A}_{\sigma}(G)$.

iii.) If $\sigma$ is submultiplicative then $\mathcal{A}_{\sigma}(G)$ is a locally multiplicatively convex algebra: the norms $\|\cdot\|_{L, \sigma, R}$ are submultiplicative.

Note that in the almost submultiplicative case the norms $\|\cdot\|_{L, \sigma, R}$ are not submultiplicative. In both cases, passing to the projective limit solved two problems at once: we get a continuous coproduct and independence on the choice of the word length.

Since we know that $\mathcal{A}_{\sigma}(G)$ is actually independent of the choice of generators we can easily discuss the relation with group morphisms. Let $G^{\prime}$ be another finitely generated group and let $\phi: G \longrightarrow G^{\prime}$ be a group morphism. We can then extend $\phi$ to a linear map $\phi: \mathbb{C}[[G]] \longrightarrow \mathbb{C}\left[\left[G^{\prime}\right]\right]$ by

$$
\phi\left(\sum_{g \in G} a_{g} \mathrm{e}_{g}\right)=\sum_{g \in G} a_{g} \mathrm{e}_{\phi(g)} .
$$

Restricted to $\mathbb{C}[G]$ this extension takes values in $\mathbb{C}\left[G^{\prime}\right]$ and yields a Hopf *-algebra morphism. If $S$ is a set of generators of $G$ as above we get a word length $L$ and we get a set $\phi(S) \subseteq G^{\prime}$ which we can extend to a set of generators $S^{\prime}$ of $G^{\prime}$. This gives a word length $L^{\prime}$ for $G^{\prime}$ which satisfies

$$
L^{\prime}(\phi(g)) \leq L(g)
$$

for $g \in G$. From this the monotonicity of $\sigma$ implies immediately the estimate

$$
\|\phi(a)\|_{L^{\prime}, \sigma, R} \leq\|a\|_{L, \sigma, R}
$$

for all $a \in \mathbb{C}[[G]]$. In particular, restricted to the Banach spaces $\ell_{L, \sigma, R}^{1}(G)$ and $\ell_{L^{\prime}, \sigma, R}^{1}(G)$ the map $\phi$ is a continuous linear operator with operator norm 1 since on the group units we get $\left\|\phi\left(\mathrm{e}_{e}\right)\right\|_{L^{\prime}, \sigma, R}=$ $1=\left\|\mathrm{e}_{e}\right\|_{L, \sigma, R}$. From this we get the following functoriality of the construction of the algebra $\mathcal{A}_{\sigma}(G)$ :

Theorem 4.5 (The algebra $\mathcal{A}_{\sigma}(G)$ ) Let $\sigma$ be an almost submultiplicative growth function. Then assigning $\mathcal{A}_{\sigma}(G)$ to a finitely generated group $G$ gives a covariant functor from the category of finitely generated groups to the category of Fréchet-Hopf ${ }^{*}$-algebras, where on morphisms we use the continuous extension as above. If in addition $\sigma$ is submultiplicative, then $\mathcal{A}_{\sigma}(G)$ is in addition locally multiplicatively convex.

Proof: The functoriality is clear and the remaining parts have been shown already.

Remark 4.6 The submultiplicative case is of course nicer as here we have the entire holomorphic calculus of locally multiplicatively convex algebras at hand, see e.g. [9]. In the almost submultiplicative case this is not true in general. In both situations we have many examples thanks to Example 3.2 .

Since $\mathcal{A}_{\sigma}(G) \subseteq \ell^{1}(G)$ is a Hopf subalgebra containing $\mathbb{C}[G]$, the group-like elements of $\mathcal{A}_{\sigma}(G)$ are again just the basis vectors $\left\{\mathrm{e}_{g}\right\}_{g \in G}$. 


\section{Comparison with Previous Results}

In this section we relate our general construction of a Fréchet-Hopf *-algebra completion of the algebraic group algebra $\mathbb{C}[G]$ to some previous and well-known constructions.

In 6,7] the polynomial growth version was studied. In our notation this corresponds to the algebra $\mathcal{A}_{\sigma}(G)$ with $\sigma$ being the linear growth $\sigma(n)=1+n$ or any other non-constant polynomial according to Lemma 3.3 the topology of $\mathcal{A}_{\sigma}$ will not depend on the particular choice of $\sigma$ as long as it is polynomial as we shall see Proposition 6.2 in some larger generality. Jolissaint then studied many properties of $\mathcal{A}_{\sigma}(G)$ and its completion including many applications to $C^{*}$-algebraic versions of the group algebra.

In [1] a general construction of seminorms for an algebra with countable vector space basis was proposed and exemplified. Among many other examples, the group algebra of a finitely generated group was discussed. We want to show that this construction fits into our general approach for a specific choice of $\sigma$. In fact, understanding the quite mysterious recursion scheme of [1] better was one of the motivations for the present paper.

Let us briefly recall the construction from [1: first we choose a function c: $G \longrightarrow \mathbb{R}^{+}$with $\mathrm{c}(g)=\mathrm{c}\left(g^{-1}\right)$. Then out of this function one defines recursively the numbers $h_{0,0, g}(a)=\left|a_{g}\right| \mathrm{c}(g)$ and

$$
h_{m+1,2 \ell, k}(a)=\sum_{g \in G} h_{m, \ell, g}(a)^{2} \frac{\mathrm{c}(k)}{\mathrm{c}(g) \mathrm{c}\left(g^{-1} k\right)} \quad \text { and } \quad h_{m+1,2 \ell+1, k}(a)=h_{m+1,2 \ell, k^{-1}}(a)
$$

for $m \in \mathbb{N}, \ell=0, \ldots, 2^{m}-1$ and $k \in G$. Then the quantities

$$
\|a\|_{m, \ell, g}(a)=\sqrt[2^{m}]{h_{m, \ell, g}(a)}
$$

turn out to be seminorms which make the product of $\mathbb{C}[G]$ continuous provided one can guarantee that $\|a\|_{m, \ell, g}<\infty$ is finite at all: this will depend on the choice of the scaling function c. While there are also other possibilities, one successful choice in [1] was to consider $\mathrm{c}(g)=(L(g) !)^{\rho}$ for a fixed value $\rho>0$. Then the countable collection (5.2) of seminorms make the group algebra a locally convex algebra which has a Fréchet algebra completion denoted by $\mathcal{A}_{L, \rho}(G)$. To relate the two topology we first show the following estimate:

Lemma 5.1 Let $\sigma(n)=n$ ! be the factorial growth and let $R, \rho>0$ be fixed. For $a \in \mathbb{C}[G]$ one has:

i.) $\left|a_{g}\right| \leq(L(g) !)^{\rho\left(2^{-m}-1\right)}\|a\|_{m+1,0, e}$ for $m \in \mathbb{N}_{0}$.

ii.) For large enough $m$ there is a constant $c>0$ such that $\|a\|_{L, \sigma, R} \leq c\|a\|_{m, 0, e}$ provided $R<\rho$.

iii.) For all $m \in \mathbb{N}_{0}$ and for all $0<\epsilon<4^{-m} \rho$ there exists a constant $c_{m, \epsilon}$ such that for all $k \in G$ one has $h_{m, \ell, k}(a) \leq c_{m, \epsilon}(L(k) !)^{4^{m} \epsilon}\|a\|_{L, \sigma, \rho-\epsilon}^{2^{m}}$.

Proof: The first claim is [1, Lem. 3.11] and follows directly from the definition and a little induction on $m$. For the second, we estimate

$$
\|a\|_{L, \sigma, R}=\sum_{g \in G}\left|a_{g}\right|(L(g) !)^{R} \leq \sum_{g \in G}(L(g) !)^{R}(L(g) !)^{\rho\left(2^{-m}-1\right)}\|a\|_{m+1,0, e}
$$

by the first part. Taking $m$ large enough, $R-\rho\left(1-2^{-m}\right)$ becomes negative. But a negative power of the function $g \mapsto L(g)$ ! is always summable over $G$, yielding the constant $c$ we need. For the third part we clearly find $c_{0, \epsilon}$ with the desired property. Thus we prove the estimate by induction on $m$. We have for $0<\epsilon<4^{-m-1} \rho<4^{-m} \rho$ and hence

$$
h_{m+1,2 \ell, k}(a) \leq \sum_{g \in G} c_{m, \epsilon}^{2}(L(g) !)^{4^{m} 2 \epsilon}\|a\|_{L, \sigma, \rho-\epsilon}^{2^{m+1}} \frac{(L(k) !)^{\rho}}{(L(g) !)^{\rho}\left(L\left(g^{-1} k\right) !\right)^{\rho}}
$$




$$
=c_{\epsilon, m}^{2}\|a\|_{L, \sigma, \rho-\epsilon}^{2^{m+1}}\left(\sum_{(1)}+\sum_{(2)}+\sum_{(3)}\right)(L(g) !)^{4^{m} 2 \epsilon} \frac{(L(k) !)^{\rho}}{(L(g) !)^{\rho}\left(L\left(g^{-1} k\right) !\right)^{\rho}},
$$

where we distinguish the following three cases for $g \in G$ in the summation: case (1) is $L(k)<L(g)$, case (2) is $L(k) \geq L(g)$ and $L(k)<L\left(g^{-1} k\right)$, and case (3) is $L(k) \geq L(g)$ and $L(k) \geq L\left(g^{-1} k\right)$. The first contribution can be estimated as

$$
\begin{aligned}
\sum_{(1)}(L(g) !)^{4^{m}} 2 \epsilon \frac{(L(k) !)^{\rho}}{(L(g) !)^{\rho}\left(L\left(g^{-1} k\right) !\right)^{\rho}} & \leq \sum_{(1)}(L(g) !)^{4^{m} 2 \epsilon} \frac{1}{\left(L\left(g^{-1} k\right) !\right)^{\rho}} \\
& \leq \sum_{h \in G}\left(\begin{array}{c}
L(k)+L(h) \\
L(h)
\end{array}\right)^{4^{m} 2 \epsilon} \frac{(L(k) !)^{4^{m} 2 \epsilon}}{(L(h) !)^{\rho-4^{m} 2 \epsilon}} \\
& \leq(L(k) !)^{4^{m} 2 \epsilon} 2^{4^{m} 2 \epsilon L(k)} \sum_{h \in G} \frac{2^{4^{m}} 2 \epsilon L(h)}{(L(h) !)^{\rho-4^{m} 2 \epsilon}}
\end{aligned}
$$

using first the substitution $h=g^{-1} k$ and then the standard estimate for a binomial coefficient. Now the last sum converges as we have a positive power of the factorial of the length in the denominator by assumption. This gives a constant independent of $k$ while the first terms can be estimated by another constant times $(L(k) !)^{4^{m+1} \epsilon}$. For the second we have

$$
\sum_{(2)}(L(g) !)^{4^{m}} 2 \epsilon \frac{(L(k) !)^{\rho}}{(L(g) !)^{\rho}\left(L\left(g^{-1} k\right) !\right)^{\rho}} \leq \sum_{g \in G} \frac{1}{(L(g) !)^{\rho-4^{m} 2 \epsilon}}<\infty,
$$

independently of $k$ since still $\rho-4^{m} 2 \epsilon>0$. Thus the second contribution can be estimated by a constant times $(L(k) !)^{4^{m+1} \epsilon}$ directly. For the third contribution we get

$$
\begin{aligned}
\sum_{(3)}(L(g) !)^{4^{m}} 2 \epsilon \frac{(L(k) !)^{\rho}}{(L(g) !)^{\rho}\left(L\left(g^{-1} k\right) !\right)^{\rho}} & \leq \sum_{(3)}(L(g) !)^{4^{m} 2 \epsilon}\left(\begin{array}{c}
L(g)+L\left(g^{-1} k\right) \\
L(g)
\end{array}\right)^{\rho} \\
& \leq 2^{4^{m+1} \epsilon L(k)} \sum_{(3)}(L(k) !)^{4^{m} 2 \epsilon}
\end{aligned}
$$

again by the usual estimate of the binomial coefficient. Now the condition $L(g) \leq L(k)$ in (3) gives at most $(2 N)^{L(k)}$ terms and hence the whole sum grows at most exponentially in $L(k)$. Thus we can also estimate this last contribution by a constant times $(L(k) !)^{4^{m+1} \epsilon}$. Putting everything together gives the third claim.

Proposition 5.2 Let $\rho>0$ and let $\sigma(n)=n$ ! be the factorial growth. For the algebra $\mathcal{A}_{L, \rho}(G)$ from [1] we have

$$
\mathcal{A}_{L, \rho}(G)=\ell_{L, \sigma, \rho^{-}}^{1}(G) .
$$

Proof: Indeed, the mutual estimates from Lemma [5.1, [ii.) and iii.), directly show that the two locally convex topologies on $\mathbb{C}[G]$ coincide. Hence the corresponding completions coincide as well.

This finally puts the rather ad-hoc and mysterious construction of [1] into a larger context. It also emphasizes the importance of the factorial growth. Note that the algebra $\mathcal{A}_{L, \rho}(G)$ will in general depend on the choice of the length functional $L$ : only after the projective limit $\rho \longrightarrow \infty$ we get independence. Note also that [1] was a much more general construction leading to non-trivial results far beyond group algebras. In fact, the construction of a locally convex topology for star product algebras was the primary goal of that work. 


\section{Properties of $\mathcal{A}_{\sigma}$}

In this section we shall investigate general properties of the functor $\mathcal{A}_{\sigma}$ and the resulting algebras: we are interested in the role of $\sigma$, the nuclearity properties, and in the topological bases of the resulting algebras.

We begin with the dependence on $\sigma$. Here we first observe the following statement:

Lemma 6.1 Let $\sigma \preccurlyeq \sigma^{\prime}$ with constants $c, k \geq 1$ as in (3.8). Without restriction we assume $c, k \in \mathbb{N}$.

i.) If $\sigma$ and $\sigma^{\prime}$ are submultiplicative then for all $R>0$ we have $\|a\|_{L, \sigma, R} \leq c^{R}\|a\|_{L, \sigma^{\prime}, c k R}$.

ii.) If $\sigma$ and $\sigma^{\prime}$ are almost submultiplicative then we have for all $R>0$ and $\epsilon>0$ a constant $c^{\prime}$ with $\|a\|_{L, \sigma, R} \leq c^{\prime}\|a\|_{L, \sigma^{\prime}, c k R+\epsilon}$.

Proof: Let $\sigma$ be almost submultiplicative and let $c_{\epsilon, c}>0$ be such that $\sigma(n m) \leq c_{\epsilon, c} \sigma(n)^{m+\epsilon}$, see (3.7). Then

$$
\|a\|_{L, \sigma, R}=\sum_{g \in G}\left|a_{g}\right| \sigma(L(g))^{R} \leq c^{R} \sum_{g \in G}\left|a_{g}\right| \sigma^{\prime}(c L(g))^{k R} \leq c^{R} c_{\epsilon, c}^{k R} \sum_{g \in G}\left|a_{g}\right| \sigma^{\prime}(L(g))^{c k R+\epsilon k R} .
$$

Rescaling $\epsilon$ and renaming the constants gives the result for the almost submultiplicative case, the submultiplicative case is even easier.

This estimate for the norms gives immediately continuous inclusions

$$
\ell_{L, \sigma^{\prime}, c k R}^{1}(G) \longrightarrow \ell_{L, \sigma, R}^{1}(G)
$$

in the submultiplicative case and analogously

$$
\ell_{L, \sigma^{\prime}, c k R+\epsilon}^{1}(G) \longrightarrow \ell_{L, \sigma, R}^{1}(G)
$$

in the almost submultiplicative case. Since we are interested in the projective limit $R \longrightarrow \infty$, the differences between the submultiplicative and the almost submultiplicative case disappear and we get the following statement:

Proposition 6.2 Let $\sigma$ and $\sigma^{\prime}$ be almost submultiplicative growth functions with $\sigma \preccurlyeq \sigma^{\prime}$. Then we have the continuous inclusion $\mathcal{A}_{\sigma^{\prime}}(G) \longrightarrow \mathcal{A}_{\sigma}(G)$. In particular, for $\sigma \sim \sigma^{\prime}$ we have

$$
\mathcal{A}_{\sigma}(G)=\mathcal{A}_{\sigma^{\prime}}(G) \text {. }
$$

In a next step we consider the basis $\left\{\mathrm{e}_{g}\right\}_{g \in G}$ of the algebraic group algebra. This turns out to be an absolute Schauder basis of $\mathcal{A}_{\sigma}(G)$ : First we recall that the topology of $\mathcal{A}_{\sigma}(G)$ is finer than the $\ell^{1}$-topology where we have constant weights in the definition of the $\ell^{1}$-norm instead of growing ones. Hence the evaluation functionals $\delta_{g}: \ell^{1}(G) \longrightarrow \mathbb{C}$ restrict to continuous linear functionals on $\mathcal{A}_{\sigma}(G)$. Moreover, we note that

$$
\left\|\mathrm{e}_{g}\right\|_{L, \sigma, R}=\sigma(L(g))^{R}
$$

for all $g \in G$ by the very definition of the norms $\|\cdot\|_{L, \sigma, R}$.

Proposition 6.3 Let $\sigma$ be an almost submultiplicative growth function. Then the vectors $\left\{\mathrm{e}_{g}\right\}_{g \in G}$ together with the evaluation functionals $\left\{\delta_{g}\right\}_{g \in G}$ form an absolute Schauder basis of $\mathcal{A}_{\sigma}(G)$.

Proof: Let $a \in \mathcal{A}_{\sigma}(G)$ and let $\|\cdot\|_{L, \sigma, R}$ be one of the defining seminorms. Then we have

$$
\sum_{g \in G}\left|\delta_{g}(a)\right|\left\|\mathrm{e}_{g}\right\|_{L, \sigma, R}=\sum_{g \in G}\left|a_{g}\right| \sigma(L(g))^{R}=\|a\|_{L, \sigma, R}
$$

which is the estimate (even equality) we need to show that we have an absolute Schauder basis, see (A.4), since clearly $a=\sum_{g \in G} a_{g} \mathrm{e}_{g}$ converges (unconditionally) in the topology of $\mathcal{A}_{\sigma}(G)$. 
Remark 6.4 Having an absolute Schauder basis is a rather strong property of a locally convex space. It follows that it is a Köthe space, see e.g. [5, Sect. 1.7.F] or [8, §27]. In our case, the Köthe matrix of $\mathcal{A}_{\sigma}(G)$ is given by the matrix

$$
K\left(\mathcal{A}_{\sigma}(G)\right)=\left(\left\|\mathrm{e}_{g}\right\|_{L, \sigma, n}\right)_{g \in G, n \in \mathbb{N}}=\left(\sigma(L(g))^{n}\right)_{g \in G, n \in \mathbb{N}},
$$

viewed as $G \times \mathbb{N}$ matrix. Many locally convex properties of $\mathcal{A}_{\sigma}(G)$ are now encoded in this matrix.

We come now to the main property of $\mathcal{A}_{\sigma}(G)$ : nuclearity. Since we have a Fréchet topology, it is reasonable to ask whether it is nuclear or not.

Lemma 6.5 For a growth function $\sigma$ with $(1+n) \preccurlyeq \sigma$ we have $\sigma_{G} \preccurlyeq \sigma$ iff $\beta_{G} \preccurlyeq \sigma$.

Proof: Since $\sigma_{G}(n) \leq \beta_{G}(n)$, one direction is trivial. Thus let $\sigma_{G} \preccurlyeq \sigma$ and hence $\sigma_{G}(n) \leq c \sigma(c n)^{k}$ for some $c, k \geq 1$. Then

$$
\beta_{G}(n)=\sum_{\ell=0}^{n} \sigma_{G}(\ell) \leq \sum_{\ell=0}^{n} c \sigma(c \ell)^{k} \leq \sum_{\ell=0}^{n} c \sigma(c n)^{k}=c(1+n) \sigma(c n)^{k},
$$

from which we get $\beta_{G} \preccurlyeq(1+n) \sigma$ But for $(1+n) \preccurlyeq \sigma$ we have $(1+n) \sigma \preccurlyeq \sigma^{2} \sim \sigma$ by (3.9) and (3.10).

It is quite reasonable to consider growth functions $\sigma$ which grow at least polynomially: a finitely generated infinite group grows at least linearly. If $(1+n) \preccurlyeq \sigma$ we get

$$
\frac{1}{\sigma} \in \ell^{p}
$$

for some $p \geq 1$ by using the very definition of the relation $\preccurlyeq$. For such growth functions, the following theorem gives a complete answer to the question of nuclearity:

Theorem 6.6 (Nuclearity of $\mathcal{A}_{\sigma}(G)$ ) Let $(1+n) \preccurlyeq \sigma$ be an almost submultiplicative growth function. Then $\mathcal{A}_{\sigma}(G)$ is nuclear iff $\beta_{G} \preccurlyeq \sigma$.

Proof: There are many ways to define nuclearity. Here we rely on the Grothendieck-Pietsch criterion for sequence spaces with absolute Schauder bases, see e.g. [8, Thm. 28.15] or [10, Sect. 6.1]: since the topology is defined by means of weighted $\ell^{1}$-norms the Grothendieck-Pietsch criterion says that $\mathcal{A}_{\sigma}(G)$ is nuclear iff for every continuous seminorm $\|\cdot\|_{L, \sigma, R}$ we find another continuous seminorm $\|\cdot\|_{L, \sigma, R^{\prime}}$ from our defining system of seminorms such that the ratio

$$
\frac{\left\|\mathrm{e}_{g}\right\|_{L, \sigma, R}}{\left\|\mathrm{e}_{g}\right\|_{L, \sigma, R^{\prime}}}=\frac{1}{\sigma(L(g))^{R^{\prime}-R}}
$$

is summable over $G$. This is clearly equivalent to say that we find a $\rho>0$ such that $\frac{1}{\sigma(L(g))^{\rho}}$ is summable over $G$, by taking $R^{\prime}$ large enough. Now first we note that $\beta_{G} \preccurlyeq \sigma$ iff $\sigma_{G} \preccurlyeq \sigma$ by Lemma 6.5. Hence we can use the more adapted surface growth $\sigma_{G}$ instead. We first assume $\sigma_{G} \preccurlyeq \sigma$ and choose $c, k>0$ with $\sigma_{G}(n) \leq c \sigma(c n)^{k}$ for all $n \in \mathbb{N}_{0}$. Since $\sigma$ is almost submultiplicative, we find for every $\epsilon>0$ a $c^{\prime}>0$ such that

$$
\sum_{g \in G} \frac{1}{\sigma(L(g))^{\rho}}=\sum_{n=0}^{\infty} \frac{\sigma_{G}(n)}{\sigma(n)^{\rho}} \leq \sum_{n=0}^{\infty} \frac{c \sigma(c n)^{k}}{\sigma(n)^{\rho}} \leq c^{\prime} \sum_{n=0}^{\infty} \frac{\sigma(n)^{c k+\epsilon}}{\sigma(n)^{\rho}}
$$


according to (3.7). If $\sigma$ is even submultiplicative, it suffices to take $\epsilon=0$ and $c^{\prime}=1$. Since by assumption $\frac{1}{\sigma} \in \ell^{p}$ for some $p \geq 1$, we can take $\rho$ large enough to see that the right hand side is summable. This shows that $\mathcal{A}_{\sigma}(G)$ is nuclear in this case. Conversely, assume $\sigma_{G} \preccurlyeq \sigma$ does not hold. By taking $c=1$ we find for all $k \in \mathbb{N}$ an index $n_{k}$ such that $\sigma_{G}\left(n_{k}\right)>\sigma\left(n_{k}\right)^{k}$. Now fix $\rho>0$ then for all $k \geq \rho$ we have a $n_{k}$ with $\sigma_{G}\left(n_{k}\right)>\sigma\left(n_{k}\right)^{\rho}$. Hence the quotient sequence $\frac{\sigma_{G}(n)}{\sigma(n)^{\rho}}$ can not be a zero sequence, let alone summable. Thus $\mathcal{A}_{\sigma}(G)$ is not nuclear.

Remark 6.7 The condition $\sigma_{G} \preccurlyeq \sigma$, which is equivalent to $\beta_{G} \preccurlyeq \sigma$ according to Lemma 6.5, can also be understood as follows: for an at least polynomially growing $\sigma$, we have $\sigma_{G} \preccurlyeq \sigma$ iff

$$
\frac{1}{L^{*} \sigma} \in \ell^{p}(G)
$$

for some $p \geq 1$, where $L$ is the word length and $L^{*} \sigma=\sigma \circ L$ is the pull-back.

Corollary 6.8 If $\sigma$ is an almost submultiplicative growth growing at least exponentially, then $\mathcal{A}_{\sigma}(G)$ is always nuclear.

Corollary 6.9 If $G$ is a group with polynomial growth then $\mathcal{A}_{\sigma}(G)$ is always nuclear.

The following particular case was treated by Jolissaint [7, Thm. 3.1.7]:

Corollary 6.10 Let $\sigma$ be the polynomial growth. Then $\mathcal{A}_{\sigma}(G)$ is nuclear iff $G$ has polynomial growth.

Yet another aspect of the condition $\beta_{G} \preccurlyeq \sigma$ is the following: in our definition of the norm $\|\cdot\|_{L, \sigma, R}$ we have chosen a $\ell^{1}$-version since this will yield good algebraic properties. However, for many other reasons other $\ell^{p}$-version might be useful as well: the $p=\infty$ case will be needed for the dual in the next section, the $\ell^{2}$-version has important applications when it comes to comparison with $C^{*}$-algebraic versions of the group algebra as discussed e.g. in [6]. For $p \geq 1$ as well as for $p=\infty$ we define

$$
\|a\|_{\ell^{p}, L, \sigma, R}=\sqrt[p]{\sum_{g \in G}\left|a_{g}\right|^{p} \sigma(L(g))^{R}} \quad \text { and } \quad\|a\|_{\ell^{\infty}, L, \sigma, R}=\sup _{g \in G}\left|a_{g}\right| \sigma(L(g))^{R} \in[0, \infty]
$$

for $a \in \mathbb{C}[[G]]$ and set

$$
\ell_{L, \sigma, R}^{p}(G)=\left\{a \in \mathbb{C}[[G]] \mid\|a\|_{\ell^{p}, L, \sigma, R}<\infty\right\} .
$$

Clearly, we obtain Banach spaces and continuous inclusions

$$
\ell_{L, \sigma, R}^{1}(G) \subseteq \ell_{L, \sigma, R}^{p}(G) \subseteq \ell_{L, \sigma, R}^{q}(G) \subseteq \ell_{L, \sigma, R}^{\infty}(G)
$$

for $p \leq q$ according to the obvious norm estimates

$$
\|a\|_{\ell^{\infty}, L, \sigma, R} \leq\|a\|_{\ell^{q}, L, \sigma, R} \leq\|a\|_{\ell^{p}, L, \sigma, R} \leq\|a\|_{\ell^{1}, L, \sigma, R}=\|a\|_{L, \sigma, R} .
$$

In general, all these topologies will be quite different. However, the nuclearity condition $\beta_{G} \preccurlyeq \sigma$ helps to simplify their mutual relations drastically:

Proposition 6.11 Let $\sigma$ be an almost submultiplicative growth function with $\beta_{G} \preccurlyeq \sigma$. Then for every $R \geq 0$ there exists a $R^{\prime}>R$ and a constant $c$ such that

$$
\|a\|_{L, \sigma, R} \leq c\|a\|_{\ell^{\infty}, L, \sigma, R^{\prime}}
$$

for all $a \in \mathbb{C}[[G]]$. 
Proof: This is essentially the same argument as needed for Theorem [6.6. Let $a \in \mathbb{C}[[G]]$. Then

$$
\|a\|_{L, \sigma, R}=\sum_{g \in G}\left|a_{g}\right| \sigma(L(g))^{R^{\prime}} \frac{1}{\sigma(L(g))^{R^{\prime}-R}} \leq\|a\|_{\ell^{\infty}, L, \sigma, R^{\prime}} \sum_{g \in G} \frac{1}{\sigma(L(g))^{R^{\prime}-R}}
$$

as an inequality in $[0,+\infty]$. If $\beta_{G} \preccurlyeq \sigma$ we know that for large enough $R^{\prime}$ the remaining series becomes finite, hence giving the constant $c$ needed for (6.12).

Together with (6.11) it follows that in the projective limit $R \longrightarrow \infty$ it is irrelevant which $\ell^{p}$-version we use to define the topology on $\mathcal{A}_{\sigma}(G)$ : all yield the same nuclear Fréchet topology.

\section{The Dual}

In this section we shall investigate the dual of $\mathcal{A}_{\sigma}(G)$ and determine some of its basic properties. Since we have an (absolute) Schauder basis, the dual can be described by the standard techniques for Köthe spaces. The basic idea is to determine the duals of the spaces $\ell_{L, \sigma, R}^{1}(G)$ and pass to the projective limit afterwards: under lucky circumstances this will be an inductive limit for the duals.

If $\Phi: \mathcal{A}_{\sigma}(G) \longrightarrow \mathbb{C}$ is a continuous linear functional, then it is uniquely determined by its values on the absolute Schauder basis $\left\{\mathrm{e}_{g}\right\}_{g \in G}$. Hence on $a \in \mathbb{C}[G] \subseteq \mathcal{A}_{\sigma}(G)$ it is given by the pairing $\Phi(a)=\langle\varphi, a\rangle$ with a unique $\varphi \in \mathbb{C}[[G]]$. However, not all $\varphi$ give a continuous extension of the pairing to a continuous linear functional on the whole algebra $\mathcal{A}_{\sigma}(G)$. Here we need to estimate the growth of the coefficients by means of the weighted supremum norm

$$
\|b\|_{\ell^{\infty}, L, \sigma,-R}=\sup _{g \in G} \frac{\left|b_{g}\right|}{\sigma(L(g))^{R}} .
$$

Note that we need to take a negative value $-R \leq 0$ of the parameter in the definition of the supremum norm (6.8).

From the general statements in Appendix $\mathrm{A}$ we know that $\ell_{L, \sigma,-R}^{\infty}(G)$ is isomorphic to the topological dual of the Banach space $\ell_{L, \sigma, R}^{1}(G)$. We shall now make this isomorphism explicit such that the bimodule structure is continuous as well. To this end, we first note that the antipode $S$ is a continuous operator on $\ell_{L, \sigma,-R}^{\infty}(G)$. In fact, we have

$$
\|S(b)\|_{\ell^{\infty}, L, \sigma,-R}=\|b\|_{\ell^{\infty}, L, \sigma,-R}
$$

since $L\left(g^{-1}\right)=L(g)$.

The continuity of the bimodule structure is controlled by the following estimates, which we have to formulate for the submultiplicative and the almost submultiplicative case separately:

Lemma 7.1 Let $a \in \mathbb{C}[G]$ and $b \in \mathbb{C}[[G]]$ and let $h \in G$. Moreover, let $R \geq 0$.

i.) For a submultiplicative growth function $\sigma$ we have

$$
\left|(a b)_{h}\right| \leq \sigma(L(h))^{R}\|a\|_{L, \sigma, R}\|b\|_{\ell^{\infty}, L, \sigma,-R} .
$$

The same estimate holds for $(b a)_{h}$.

ii.) For an almost submultiplicative growth function $\sigma$ we have for all $\epsilon>0$ a constant $c>0$ such that

$$
\left|(a b)_{h}\right| \leq c \sigma(L(h))^{R+\epsilon}\|a\|_{L, \sigma, R+\epsilon}\|b\|_{\ell \infty, L, \sigma,-R} .
$$

The same estimate holds for $(b a)_{h}$. 
Proof: We prove the second statement, the first is analogous but simpler. We fix $\epsilon>0$ and choose $c \geq 1$ such that $\sigma(n+m) \leq c \sigma(n)^{1+\epsilon} \sigma(m)^{1+\epsilon}$. Then we get

$$
\begin{aligned}
\left|(a b)_{h}\right| & =\left|\sum_{g \in G} a_{g} \sigma\left(L\left(g^{-1} h\right)\right)^{R} \frac{b_{g^{-1} h}}{\sigma\left(L\left(g^{-1} h\right)\right)^{R}}\right| \\
& \leq \sum_{g \in G}\left|a_{g}\right| c \sigma(L(g))^{R(1+\epsilon)} \sigma(L(h))^{R(1+\epsilon)} \frac{\left|b_{g^{-1} h}\right|}{\sigma\left(L\left(g^{-1} h\right)\right)^{R}} \\
& \leq c \sigma(L(h))^{R(1+\epsilon)}\|a\|_{L, \sigma, R(1+\epsilon)} \sup _{k \in G} \frac{\left|b_{k}\right|}{\sigma(L(k))^{R}} .
\end{aligned}
$$

Since this can be done for all $\epsilon>0$, a simple rescaling gives (17.4).

Remarkably, for $h=e$ the estimate (17.4) can be improved to

$$
\left|(a b)_{e}\right| \leq\|a\|_{L, \sigma, R}\|b\|_{\ell, L, \sigma,-R}
$$

also for the almost submultiplicative case by a direct estimate. For general $h$ however, we get only the weaker estimate from (7.4). Based on these estimates the following proposition is now a simple consequence of the algebraic properties of the trace functional tr outlined in Section 2.

Proposition 7.2 Let $R \geq 0$ and let $\sigma$ be an almost submultiplicative growth function. Then the map

$$
\ell_{L, \sigma,-R}^{\infty}(G) \ni b \mapsto\langle S(b), \cdot\rangle=\operatorname{tr}(b \cdot) \in \ell_{L, \sigma, R}^{1}(G)^{\prime}
$$

is an isometric isomorphism of Banach spaces.

Proof: From the general results on the dual of weighted $\ell^{1}$-spaces as in the Appendix $\AA$ and the algebraic considerations from Section 2 this follows now from the estimate in (7.5).

While (7.6) is always an isomorphism of Banach spaces, the situation for the bimodule structures is slightly more complicated. Here we have to distinguish the submultiplicative and the almost submultiplicative case. For a submultiplicative $\sigma$ we know that $\ell_{L, \sigma, R}^{1}(G)$ is a Banach algebra and hence its dual is a Banach bimodule over it in a canonical way. For the almost submultiplicative case we first have to pass from $\ell_{L, \sigma, R}^{1}(G)$ to the projective limit $\ell_{L, \sigma, R^{-}}^{1}(G)$ in order to get an algebra at all. Ultimately, we want to consider $R \longrightarrow \infty$ for both cases.

To this end we compare the different $\ell^{\infty}$-norms: obviously we have

$$
\|b\|_{\ell \infty, L, \sigma,-R} \leq\|b\|_{\ell^{\infty}, L, \sigma,-R^{\prime}}
$$

for $R \geq R^{\prime} \geq 0$. Moreover, for $R=0$ we have the usual supremum norm. This results in continuous inclusions

$$
\ell_{L, \sigma, R}^{1} \longrightarrow \ell^{1}(G) \longrightarrow \ell^{\infty}(G) \longrightarrow \ell_{L, \sigma,-R^{\prime}}^{\infty}(G) \longrightarrow \ell_{L, \sigma,-R}^{\infty}(G)
$$

for $R \geq R^{\prime} \geq 0$.

Proposition 7.3 Let $\sigma$ be a submultiplicative growth function and let $R \geq 0$.

i.) For $a \in \mathbb{C}[G]$ and $b \in \mathbb{C}[[G]]$ one has

$$
\|a b\|_{\ell^{\infty}, L, \sigma,-R} \leq\|a\|_{L, \sigma, R}\|b\|_{\ell^{\infty}, L, \sigma,-R} \quad \text { as well as } \quad\|b a\|_{\ell^{\infty}, L, \sigma,-R} \leq\|a\|_{L, \sigma, R}\|b\|_{\ell^{\infty}, L, \sigma,-R} .
$$

ii.) The bimodule structure of $\mathbb{C}[[G]]$ with respect to the group algebra $\mathbb{C}[G]$ restricts to a continuous bimodule structure on $\ell_{L, \sigma,-R}^{\infty}(G)$ with respect to the Banach algebra $\ell_{L, \sigma, R}^{1}(G)$. 
iii.) The canonical inclusion $\ell_{L, \sigma, R}^{1}(G) \subseteq \ell_{L, \sigma,-R}^{\infty}(G)$ is a continuous bimodule morphism.

Proof: The first part is just Lemma 7.1. This implies the second part and hence the third part follows from the general algebraic considerations from Section 2.

Corollary 7.4 Let $\sigma$ be a submultiplicative growth function. Then for all $R \geq 0$ the algebra $\ell_{L, \sigma, R}^{1}(G)$ embeds as bimodule into its topological dual via (7.6) in a continuous way.

Indeed, the canonical bimodule structures on the dual correspond to the bimodule structures on $\ell_{L, \sigma,-R}^{\infty}(G)$ as both are the restrictions of the bimodule structures of $\mathbb{C}[[G]]$ and $\mathbb{C}[G]^{*}$ with respect to $\mathbb{C}[G]$ which then can be extended by continuity from $\mathbb{C}[G]$ to $\ell_{L, \sigma, R}^{1}(G)$.

In a next step we pass to the projective limit $\mathcal{A}_{\sigma}(G)$ of the algebras $\ell_{L, \sigma, R}^{1}(G)$ for $R \longrightarrow \infty$. First we note that the projective system is reduced, i.e. the projective limit $\mathcal{A}_{\sigma}(G)$ is dense in all the spaces $\ell_{L, \sigma, R}^{1}(G)$ :

Lemma 7.5 Let $\sigma$ be an almost submultiplicative growth function. Then the projective system of Banach spaces $\left\{\ell_{L, \sigma, R}^{1}(G)\right\}_{R \geq 0}$ is reduced.

Proof: Indeed, the subspace $\mathbb{C}[G] \subseteq \mathcal{A}_{\sigma}(G)$ is already dense in each $\ell_{L, \sigma, R}^{1}(G)$.

It follows that the dual space of $\mathcal{A}_{\sigma}(G)$ is given by the inductive limit of the dual spaces $\ell_{L, \sigma, R}^{1}(G)^{\prime}$ as a vector space, i.e. we have

$$
\mathcal{A}_{\sigma}(G)^{\prime}=\left(\operatorname{proj}_{R \longrightarrow \infty} \lim _{L, \sigma, R}(G)\right)^{\prime}=\operatorname{ind}_{R \longrightarrow \infty} \lim _{L, \sigma, R}^{1}(G)^{\prime} \cong \bigcup_{R \geq 0} \ell_{L, \sigma,-R}^{\infty}(G)
$$

as vector spaces, see e.g. [3, §26, Satz 1.6]. Note however, without more detailed information this is just an isomorphism of vector spaces, the corresponding locally convex inductive limit of the right hand side can still be rather complicated. In order to determine the locally convex topology coming from this inductive limit structure, we need the following observation:

Lemma 7.6 Let $\sigma$ be an almost submultiplicative growth function with $\beta_{G} \preccurlyeq \sigma$. Then the projective system $\left.\ell_{L, \sigma, R}^{1}(G)\right\}_{R \geq 0}$ is nuclear and hence compact.

Proof: This is essentially the Grothendieck-Pietsch criterion: we have to show that for every $R \geq 0$ there is a $R^{\prime}>R$ such that the inclusion map $\ell_{L, \sigma, R^{\prime}}^{1}(G) \longrightarrow \ell_{L, \sigma, R}^{1}(G)$ is nuclear. This inclusion map can be written as

$$
a \mapsto \sum_{g \in G} \frac{1}{\sigma(L(g))^{R^{\prime}-R}} \frac{\mathrm{e}_{g}}{\sigma(L(g))^{R}} \sigma(L(g))^{R^{\prime}} \delta_{g}(a),
$$

where we view $\sigma(L(g))^{R^{\prime}} \delta_{g}: \ell_{L, \sigma, R^{\prime}}^{1}(G) \longrightarrow \mathbb{C}$ as a continuous linear functional of functional norm 1 and where $\frac{\mathrm{e}_{g}}{\sigma(L(g))^{R}} \in \ell_{L, \sigma, R}^{1}(G)$ is a unit vector. The fact that $(*)$ is nuclear is thus a consequence of the summability of the pre-factors for large enough $R^{\prime}-R$, which is the case if $\sigma_{G} \preccurlyeq \sigma$ by the very argument used in the proof of Theorem 6.6. Finally, we use that a nuclear map is always compact.

For a compact and reduced projective system the algebraic identification (17.10) can be turned into a topological identification if one uses the strong topology on the dual, see [3, §26, Satz 2.4]:

Theorem 7.7 (The dual of $\mathcal{A}_{\sigma}(G)$ ) Let $\sigma$ be an almost submultiplicative growth function with $\beta_{G} \preccurlyeq$ $\sigma$. Then the locally convex inductive limit topology on the inductive system $\left\{\ell_{L, \sigma, R}^{1}(G)^{\prime}\right\}_{R \geq 0}$ yields the strong topology on the dual of $\mathcal{A}_{\sigma}(G)$, i.e. we have

$$
\mathcal{A}_{\sigma}(G)^{\prime}=\operatorname{ind}_{R \longrightarrow \infty} \lim _{L, \sigma, R}(G)^{\prime} \cong \operatorname{ind}_{R \longrightarrow \infty} \lim _{L, \sigma,-R}(G)
$$

in the sense of the identification (7.10) with the strong topology on the dual. 
Remark 7.8 Under the assumption $\beta_{G} \preccurlyeq \sigma$, which we found already very useful in Theorem 6.6 we have on the one hand a rather easy description of the dual space of $\mathcal{A}_{\sigma}(G)$ as vector space, namely the union of all the spaces $\ell_{L, \sigma,-R}^{\infty}(G)$. Recall that the locally convex inductive limit topology is determined by all the seminorms p on $\bigcup_{R \geq 0} \ell_{L, \sigma,-R}^{\infty}(G)$ such that the restriction of $\mathrm{p}$ to any of the individual spaces $\ell_{L, \sigma,-R}^{\infty}(G)$ is continuous. In general, it is quite hard to write down an explicit characterization of such seminorms. Nevertheless, the locally convex inductive limit topology has the nice universal property, that a linear map $\Phi: \bigcup_{R \geq 0} \ell_{L, \sigma,-R}^{\infty}(G) \longrightarrow V$ into some other locally convex space $V$ is continuous iff each restriction $\left.\Phi\right|_{\ell_{L, \sigma,-R}^{\infty}(G)}$ is continuous. On the other hand, the strong topology on the dual $\mathcal{A}_{\sigma}(G)$ is determined by the explicit seminorms $\mathrm{p}_{B}(\varphi)=\sup _{a \in B}|\varphi(a)|$ where $B \subseteq \mathcal{A}_{\sigma}(G)$ ranges over all bounded subsets of $\mathcal{A}_{\sigma}(G)$. From the general theory we know that the strong topology is complete and not just sequentially complete (as e.g. the weak* topology). It is the combination of the two aspects which makes the dual of $\mathcal{A}_{\sigma}(G)$ manageable. Note however, that the inductive limit in (7.10) will not be a strict inductive limit.

While in the Banach space situation the dual of an algebra carries a continuous bimodule structure, the bimodule structure in the general locally convex case might no longer be continuous. We recall the following well-known statement:

Proposition 7.9 Let $\mathcal{A}$ be a locally convex algebra and equip its dual $\mathcal{A}^{\prime}$ with the strong topology. Then $\mathcal{A}^{\prime}$ is a bimodule over $\mathcal{A}$ via (2.3) such that the bimodule structure is separately continuous.

Note that in the Banach space situation the strong topology is the usual norm topology on the dual. Thus this statement generalizes the results from Proposition 7.3. Since $\mathcal{A}_{\sigma}(G)$ is included continuously into $\ell^{1}(G)$ and hence, via (7.8), into the dual $\mathcal{A}_{\sigma}(G)^{\prime}$, we obtain the following statement:

Corollary 7.10 Let $\sigma$ be an almost submultiplicative growth function with $\beta_{G} \preccurlyeq \sigma$. Then $\mathcal{A}_{\sigma}(G)$ is continuously included into its strong dual $\mathcal{A}_{\sigma}(G)^{\prime}$ as a bimodule.

\section{The Spaces $\mathfrak{c}_{L, \sigma,-R}^{0}(G)$}

For fixed $R$ we have the Banach spaces $\ell_{L, \sigma,-R}^{\infty}(G)$, which constitute the topological duals of $\ell_{L, \sigma, R}^{1}(G)$, and the evaluation functionals $\delta_{g}=\operatorname{tr}\left(S\left(\mathrm{e}_{g}\right) \cdot\right)$ for $g \in G$ are continuous linear functionals, i.e. elements of the topological dual. Moreover, elements in $\ell_{L, \sigma,-R}^{\infty}(G)$ are certain formal series in the basis vectors $\mathrm{e}_{g}$. Thus it is reasonable to ask whether these vectors still form a topological basis of $\ell_{L, \sigma,-R}^{\infty}(G)$. This is not true for general reasons but the failure of being a topological basis is not too bad: we have to take into account all spaces $\ell_{L, \sigma,-R}^{\infty}(G)$ for $R \longrightarrow \infty$ in the end and we shall show in this section that between any two of them is a space $\mathfrak{c}_{L, \sigma,-R}^{0}(G)$ for which the basis vectors indeed form an unconditional Schauder basis.

As for general weighted $\ell^{\infty}$-spaces we define the following subset of $\ell_{L, \sigma,-R}^{\infty}(G)$

$$
\mathfrak{c}_{L, \sigma,-R}^{0}(G)=\left\{b \in \mathbb{C}[[G]] \mid \text { for all } \epsilon>0 \text { there is a finite subset } K \subseteq G \text { with } \sup _{g \in G \backslash K} \frac{\left|b_{g}\right|}{\sigma(L(g))^{R}}<\epsilon\right\},
$$

see also (A.6). The idea is that this space corresponds to the usual sequence space of zero sequences $\mathfrak{c}^{0}$ equipped with the supremum norm, viewed as a closed subspace of $\ell^{\infty}$. Now the sequences do of course not converge to 0 but require the weights for the estimate of being small outside a finite subset. From the general theory we obtain the following important statement:

Proposition 8.1 Let $R \geq 0$.

i.) With respect to the norm $\|\cdot\|_{\ell^{\infty}, L, \sigma,-R}$ the set $\mathfrak{c}_{L, \sigma,-R}^{0}(G)$ is a closed subspace of $\ell_{L, \sigma,-R}^{\infty}(G)$. 
ii.) The $\mathbb{C}$-span of the vectors $\left\{\mathrm{e}_{g}\right\}_{g \in G}$ is dense in $\mathfrak{c}_{L, \sigma,-R}^{0}(G)$.

iii.) The vectors $\left\{\mathrm{e}_{g}\right\}_{g \in G}$ form an unconditional Schauder basis in $\mathfrak{c}_{L, \sigma,-R}^{0}(G)$.

iv.) For $R^{\prime}>R$ we have the continuous inclusion

$$
\ell_{L, \sigma,-R}^{\infty}(G) \subseteq \mathfrak{c}_{L, \sigma,-R^{\prime}}^{0}(G)
$$

with dense image.

Proof: The first three statements are true in general and folklore, see Appendix A. For the last statement, we already know that $\ell_{L, \sigma,-R}^{\infty}(G) \subseteq \ell_{L, \sigma,-R^{\prime}}^{\infty}(G)$ is a continuous inclusion. Now let $b \in$ $\ell_{L, \sigma,-R}^{\infty}(G)$ be given. Then for all $g \in G$ we have

$$
\frac{\left|b_{g}\right|}{\sigma(L(g))^{R^{\prime}}}=\frac{\left|b_{g}\right|}{\sigma(L(g))^{R}} \frac{1}{\sigma(L(g))^{R^{\prime}-R}} \leq\|b\|_{\ell^{\infty}, L, \sigma,-R} \frac{1}{\sigma(L(g))^{R^{\prime}-R}} .
$$

Since $\sigma$ is unbounded and monotonic by definition of a growth function, the sequence $\left(\frac{1}{\sigma(n)}\right)_{n \in \mathbb{N}_{0}}$ is a monotonic zero sequence. Moreover, for every $n \in \mathbb{N}_{0}$ there are only finitely many group elements $g \in G$ with $L(g)=n$, namely $\sigma_{G}(n)$ many. Hence for $\epsilon>0$ the subset

$$
K_{\epsilon}=\left\{g \in G \mid \frac{1}{\sigma(L(g))^{R^{\prime}-R}} \geq \epsilon\right\}
$$

is finite. This shows that for $g \in G \backslash K_{\epsilon}$ we have $\frac{\left|b_{g}\right|}{\sigma(L(g))^{R^{\prime}}}<\epsilon\|b\|_{\ell^{\infty}, L, \sigma,-R}$. From here we can conclude (8.2). Since already $\mathbb{C}[G]$ is dense in $\mathfrak{c}_{L, \sigma,-R^{\prime}}^{0}(G)$, the density of $\ell_{L, \sigma,-R}^{\infty}(G)$ follows, too.

In particular, the inductive limits of the Banach spaces $\mathfrak{c}_{L, \sigma,-R}^{0}(G)$ and of the Banach spaces $\ell_{L, \sigma,-R}^{\infty}(G)$ for $R \longrightarrow \infty$ coincide. Hence it will sometimes be advantageous to replace the $\ell_{L, \sigma,-R}^{\infty}(G)$ by the easier $\mathfrak{c}_{L, \sigma,-R}^{0}(G)$ where we can use e.g. the existence of an unconditional Schauder basis.

Concerning the algebraic structure we have to restrict again to the submultiplicative case since only here we have an algebra structure for $\ell_{L, \sigma, R}^{1}(G)$. Hence it makes sense to ask whether $\mathfrak{c}_{L, \sigma,-R}^{0}(G) \subseteq$ $\ell_{L, \sigma,-R}^{\infty}(G)$ is a sub bimodule. Indeed, this is the case:

Theorem 8.2 (The bimodule $\mathfrak{c}_{L, \sigma,-R}^{0}(G)$ ) Let $\sigma$ be a submultiplicative growth function. Then $\mathfrak{c}_{L, \sigma,-R}^{0}(G)$ is a sub bimodule of $\ell_{L, \sigma,-R}^{\infty}(G)$ over $\ell_{L, \sigma, R}^{1}(G)$.

Proof: Let $b \in \mathfrak{c}_{L, \sigma,-R}^{0}(G)$ and $a \in \ell_{L, \sigma, R}^{1}(G)$ be given. We have to show that $a b, b a \in \mathfrak{c}_{L, \sigma,-R}^{0}(G)$. Let $\epsilon>0$ and fix a finite subset $K \subseteq G$ with $\frac{\left|b_{g}\right|}{\sigma(L(g))^{R}}<\epsilon$ for $g \in G \backslash K$. Moreover, let $\tilde{K} \subseteq G$ be a finite subset such that

$$
\sum_{g \in G \backslash \tilde{K}}\left|a_{g}\right| \sigma(L(g))^{R}<\epsilon
$$

which we can arrange since the series $\|a\|_{L, \sigma, R}<\infty$ converges (absolutely). Finally, let $K^{\prime} \subseteq G$ be the subset of those elements $h$ for which there exists a $g \in \tilde{K}$ with $g^{-1} h \in K$. Again, this subset $K^{\prime}$ is finite. Now for $h \in G \backslash K^{\prime}$ we estimate

$$
\begin{aligned}
\frac{\left|(a b)_{h}\right|}{\sigma(L(h))^{R}} & \leq \sum_{g \in G}\left|a_{g}\right| \frac{\sigma\left(L\left(g^{-1} h\right)\right)^{R}}{\sigma(L(h))^{R}} \frac{\left|b_{g^{-1} h}\right|}{\sigma\left(L\left(g^{-1} h\right)\right)^{R}} \\
& \leq \sum_{g \in G}\left|a_{g}\right| \sigma(L(g))^{R} \frac{\left|b_{g^{-1} h}\right|}{\sigma\left(L\left(g^{-1} h\right)\right)^{R}} \\
& \leq \sum_{g \in G, g^{-1} h \in K}\left|a_{g}\right| \sigma(L(g))^{R}\|b\|_{\ell \infty, L, \sigma,-R}+\sum_{g \in G, g^{-1} h \in G \backslash K}\left|a_{g}\right| \sigma(L(g))^{R} \epsilon
\end{aligned}
$$




$$
\begin{aligned}
& \leq\|b\|_{\ell^{\infty}, L, \sigma,-R} \sum_{g \in G \backslash \tilde{K}}\left|a_{g}\right| \sigma(L(g))^{R}+\epsilon\|a\|_{L, \sigma, R} \\
& \leq \epsilon\left(\|b\|_{\ell^{\infty}, L, \sigma,-R}+\|a\|_{L, \sigma, R}\right) .
\end{aligned}
$$

Rescaling $\epsilon$ gives the result that outside of a finite subset the coefficient $\frac{\left|(a b)_{h}\right|}{\sigma(L(h))^{R}}$ is less than $\epsilon$. But this means $a b \in \mathfrak{c}_{L, \sigma,-R}^{0}(G)$. The argument for $b a$ is analogous.

Remark 8.3 In the almost submultiplicative case things are less easy. Here we first have to pass to a projective limit $\ell_{L, \sigma, R^{-}}^{1}(G)$ in order to have an algebra at all. Then this requires an inductive limit $\ell_{L, \sigma,-R^{-}}^{\infty}(G)$ to get a bimodule structure and the dual. Inside this, one can consider the inductive limit $\mathfrak{c}_{L, \sigma,-R^{-}}^{0}(G)$. Repeating the above estimates shows that this is indeed a sub bimodule.

Remark 8.4 Since $\mathcal{A}_{\sigma}(G) \subseteq \ell_{L, \sigma, R}^{1}(G)$ is a subalgebra, it follows that all the subspaces $\mathfrak{c}_{L, \sigma, R}^{0}(G)$ of the dual $\mathcal{A}_{\sigma}(G)^{\prime}$ are sub bimodules over $\mathcal{A}_{\sigma}(G)$. Hence the dual of $\mathcal{A}_{\sigma}(G)$ has a quite rich sub bimodule structure.

\section{An Application: The Complete Growth}

Recall that the complete growth function of $G$ with respect to a chosen word length is defined by

$$
F_{L}(z)=\sum_{g \in G} \mathrm{e}_{g} z^{L(g)}=\sum_{n=0}^{\infty}\left(\sum_{g \in G, \sigma_{G}(g)=n} \mathrm{e}_{g}\right) z^{n} \in \mathbb{Z}[G][[z]],
$$

see [4] where many non-trivial properties of the complete growth were studied.

In general, this will be just a formal power series in $z$. The purpose of this section is to find an analytic scenario where we can make sense out of the convergence of $F_{L}(z)$. The first try would be to look at our algebras $\ell_{L, \sigma, R}^{1}(G)$ for a suitable choice of $\sigma$ and $R$. However, the coefficients in $F_{L}(z)$ consist of sums of group elements without any decaying pre-factors. Hence we do not expect to have reasonable convergence properties in the topologies of $\ell_{L, \sigma, R}^{1}(G)$, let alone in $\mathcal{A}_{\sigma}(G)$ where we have to require a fast decay, controlled by $\sigma$.

Thus the next idea is to consider $F_{L}(z)$ as an element in the dual of $\mathcal{A}_{\sigma}(G)$ : here we can have a not to fast growth of the coefficients. Again, this supports the point of view to think of the duality between test functions $\mathcal{A}_{\sigma}(G)$ and distributions and hence of a convergence of (9.1) in a distributional sense. Under the nuclearity assumption this turns out to be the case:

Theorem 9.1 (Convergence of the complete growth) Let $(1+n) \preccurlyeq \sigma$ be an almost submultiplicative growth function. Then the following statements are equivalent:

i.) We have $\beta_{G} \preccurlyeq \sigma$.

ii.) There exists an $R \geq 0$ such that $F_{L}(z)$ converges absolutely for $|z| \leq 1$ with respect to $\|\cdot\|_{\ell^{\infty}, L, \sigma,-R}$.

Proof: Clearly it suffices to consider $|z|=1$. The absolute convergence of the series (9.1) with respect to the norm $\|\cdot\|_{\ell^{\infty}, L, \sigma,-R}$ for $|z|=1$ means that

$$
\sum_{g \in G}\left\|\mathrm{e}_{g} z^{L(g)}\right\|_{\ell^{\infty}, L, \sigma,-R}=\sum_{g \in G} \frac{1}{\sigma(L(g))^{R}}<\infty .
$$

But this is equivalent to say that $g \mapsto \frac{1}{\sigma(L(g))}$ is $\ell^{p}$-summable over $G$ for some $p \geq 1$, namely $p=R$. By Remark 6.7 this is equivalent to $\sigma_{G} \preccurlyeq \sigma$ and hence $\beta_{G} \preccurlyeq \sigma$, by Lemma 6.5.

Corollary 9.2 For an almost submultiplicative growth function $\sigma$ with $\beta_{G} \preccurlyeq \sigma$ the complete growth $F_{L}$ is a holomorphic function of $z$ on the unit disc taking values in the strong dual of $\mathcal{A}_{\sigma}(G)$. 


\section{A Some Locally Convex Analysis}

In this short appendix we collect some basic and well-known results from locally convex analysis. More details can be found in e.g. [3, 5 .

We make extensive use of the tensor product of seminorms: if $\mathrm{p}$ and $\mathrm{q}$ are seminorms on vector spaces $V$ and $W$, respectively, then $\mathrm{p} \otimes \mathrm{q}$ defined by

$$
(\mathrm{p} \otimes \mathrm{q})(z)=\inf \left\{\sum_{i} \mathrm{p}\left(v_{i}\right) \mathrm{q}\left(w_{i}\right) \mid z=\sum_{i} v_{i} \otimes w_{i}\right\}
$$

is a seminorm on the tensor product $V \otimes W$ which is a norm iff p and q are norms. On factorizing tensors one has $(\mathrm{p} \otimes \mathrm{q})(v \otimes w)=\mathrm{p}(v) \mathrm{q}(w)$.

Very often we need seminorms build from $\ell^{1}$-like norms with respect to certain vector space bases and weighted counting measures. For their tensor product one has the following result:

Lemma A.1 Let $V=\mathbb{C}-\operatorname{span}\left\{e_{i}\right\}_{i \in I}$ and $W=\mathbb{C}-\operatorname{span}\left\{f_{j}\right\}_{j \in J}$ be vector spaces with bases. Moreover, let $\mu_{i} \geq 0$ and $\nu_{j} \geq 0$ for $i \in I$ and $j \in J$ be given and consider the $\ell^{1}$-seminorms

$$
\|v\|_{\ell^{1}(\mu)}=\sum_{i \in I}\left|v_{i}\right| \mu_{i} \quad \text { and } \quad\|w\|_{\ell^{1}(\nu)}=\sum_{j \in J}\left|w_{j}\right| \nu_{j}
$$

where $v=\sum_{i \in I} v_{i} e_{i}$ and $w=\sum_{j \in J} w_{j} f_{j}$. Then $\|\cdot\|_{\ell^{1}(\mu)} \otimes\|\cdot\|_{\ell^{1}(\nu)}=\|\cdot\|_{\ell^{1}(\mu \times \nu)}$, where

$$
\|z\|_{\ell^{1}(\mu \times \nu)}=\sum_{i \in I, j \in J}\left|z_{i j}\right| \mu_{i} \nu_{j}
$$

for $z=\sum_{i \in I, j \in J} z_{i j} e_{i} \otimes f_{j} \in V \otimes W$.

Note that for $\|\cdot\|_{\ell^{1}(\mu)}$ to be a norm we have to require $\mu_{i}>0$ for $i \in I$.

In all our constructions the existence of topological bases simplified the arguments in a substantial way. Recall that a linearly independent set $\left\{\mathrm{e}_{i}\right\}_{i \in I}$ in a locally convex space $V$ is called an unconditional Schauder basis if there are continuous coefficient functionals $\delta_{i} \in V^{\prime}$ such that for every $v \in V$ there are only countably many $\delta_{i}(v)$ different from zero and $v=\sum_{i \in I} \delta_{i}(v) \mathrm{e}_{i}$ converges: since we have not chosen an ordering of $I$ the convergence is necessarily unconditional. The Schauder basis is called absolute if for every continuous seminorm p on $V$ there is another continuous seminorm q such that

$$
\sum_{i \in I}\left|\delta_{i}(v)\right| \mathrm{p}\left(\mathrm{e}_{i}\right) \leq \mathrm{q}(v) .
$$

Note that an absolute Schauder basis is necessarily unconditional but the converse is not true in general.

Example A.2 A quite archetypal example of a Banach space with an absolute Schauder space is the sequence space $\ell^{1}$. Slightly more general, we consider a vector space $V$ with a basis $\left\{\mathrm{e}_{i}\right\}_{i \in I}$ and weights $\mu_{i}>0$ for $i \in I$ as we did that in Lemma A.1. Then the completion $\widehat{V}$ of $V$ with respect to the weighted $\ell^{1}$-norm $\|\cdot\|_{\ell^{1}(\mu)}$ can be identified with the set $\widehat{V}$ of formal series $v=\sum_{i \in I} v_{i} \mathrm{e}_{i}$ where for each $v$ at most countably many $v_{i}$ are different from zero and $\|v\|_{\ell^{1}(\mu)}=\sum_{i \in I}\left|v_{i}\right| \mu_{i}$ is finite. In more measure-theoretic terms the completion is just the space $\ell^{1}(I, \mu)$ where the index set $I$ is equipped with the weighted counting measure $\mu$. For this example it is now easy to see that the evaluation functionals $\delta_{i}: v \mapsto v_{i}$ are continuous with respect to $\|\cdot\|_{\ell^{1}(\mu)}$. In fact, they have functional norm $\left\|\delta_{i}\right\|=\frac{1}{\mu_{i}}$. Moreover, the former vector space basis $\left\{\mathrm{e}_{i}\right\}_{i \in I}$ of $V$ becomes now an absolute Schauder basis of its completion. 
We also need a concrete description of the duals of weighted $\ell^{1}$-spaces. Thus let again $V$ be a vector space with basis $\left\{\mathrm{e}_{i}\right\}_{i \in I}$ and weights $\mu_{i}>0$ which we assume to be strictly positive to be in a Banach space situation for the completion $\widehat{V}$ with respect to the norm $\|\cdot\|_{\ell^{1}(\mu)}$. If $\Phi: \widehat{V} \longrightarrow \mathbb{C}$ is a continuous linear functional then it is completely determined by its values $\Phi\left(e_{i}\right)$ on the absolute Schauder basis. If $\|\Phi\|$ is the functional norm of $\Phi$ then $\left|\Phi\left(e_{i}\right)\right| \leq\|\Phi\|\left\|e_{i}\right\|_{\ell^{1}(\mu)}=\|\Phi\| \mu_{i}$ and hence $\|\Phi\| \leq \sup _{i \in I} \frac{\left|\Phi\left(e_{i}\right)\right|}{\mu_{i}}$. In fact, it is easy to see that we have equality here. Moreover, given a linear functional on $V$, then it extends continuously to the completion (necessarily in a unique way) iff this supremum is finite. Thus we have

$$
\widehat{V}^{\prime}=\left\{\Phi \in \widehat{V}^{*} \mid \sup _{i \in I} \frac{\left|\Phi\left(\mathrm{e}_{i}\right)\right|}{\mu_{i}}<\infty\right\}
$$

and the functional norm is this above supremum. Note that this condition can also be understood as the usual supremum-norm condition with respect to the unit vectors $\frac{1}{\mu_{i}} \mathrm{e}_{i}$. Nevertheless, for our application the basis vectors $e_{i}$ will play a more important role as we will have many weighted counting measures $\mu$ simultaneously but keep the vectors $\mathrm{e}_{i}$ in our application. Hence there is no point in rescaling the vectors $\mathrm{e}_{i}$ to turn them into unit vectors for one of the measures $\mu$.

In general, the linear span of the coefficient functionals $\delta_{i}$ will not be dense in $\ell^{\infty}(I, \mu)$. Instead, its completion is a much smaller subspace corresponding to the space of zero sequences $\mathfrak{c}^{0}$. In general, one considers

$$
\mathfrak{c}^{0}(I, \mu)=\left\{\Phi \in \ell^{\infty}(I, \mu) \mid \text { for all } \epsilon>0 \text { there is a finite subset } K \subseteq I \text { with } \sup _{i \in I \backslash K} \frac{1}{\mu_{i}}\left|\Phi\left(\mathrm{e}_{i}\right)\right|<\epsilon\right\} .
$$

In general, this will be a proper subset of $\ell^{\infty}(I, \mu)$.

Lemma A.3 Assume $I$ is countable. The set $\mathfrak{c}^{0}(I, \mu) \subseteq \ell^{\infty}(I, \mu)$ is a closed subspace and the span of the evaluation functionals $\left\{\delta_{i}\right\}_{i \in I}$ is dense in $\mathfrak{c}^{0}(I, \mu)$.

If $I$ is not countable, then we still can obtain the completion of the span of the evaluation functionals where we have to add the condition to the definition of $\mathfrak{c}^{0}(I, \mu)$ that at most countably many $\Phi\left(\mathrm{e}_{i}\right)$ are different from zero. In any case, in this work we only need countable index sets $I$.

\section{References}

[1] Beiser, S., Waldmann, S.: Fréchet algebraic deformation quantization of the Poincaré disk. Crelle's J. reine angew. Math. 688 (2014), 147-207. 3, 11, 12

[2] DE LA Harpe, P.: Topics in geometric group theory. Chicago Lectures in Mathematics. University of Chicago Press, Chicago, 2000. 5, 6, 07

[3] Floret, K., Wloka, J.: Einführung in die Theorie der lokalkonvexen Räume, vol. 56 in Lecture Notes in Mathematics. Springer-Verlag, Berlin, 1968. 18,22

[4] Grigorchuk, R., Nagnibeda, T.: Complete growth functions of hyperbolic groups. Invent. Math. 130.1 (1997), 159-188. 3, 21

[5] Jarchow, H.: Locally Convex Spaces. B. G. Teubner, Stuttdart, 1981. 14, 22

[6] Jolissaint, P.: K-theory of reduced $C^{*}$-algebras and rapidly decreasing functions on groups. K-Theory 2.6 (1989), 723-735. [3, 11, 15

[7] Jolissaint, P.: Rapidly decreasing functions in reduced $C^{*}$-algebras of groups. Trans. Amer. Math. Soc. 317.1 (1990), 167-196. 3, 11, 15] 
[8] Meise, R., Vogt, D.: Einführung in die Funktionalanalysis. Vieweg-Verlag, Braunschweig, Wiesbaden, 1992. 14

[9] Michael, E. A.: Locally Multiplicatively-Convex Topological Algebras. Mem. Amer. Math. Soc. no. 11. AMS, Providence, R. I., 1952. 10

[10] Pietsch, A.: Nuclear locally convex spaces, vol. 66 in Ergebnisse der Mathematik und ihrer Grenzgebiete. Springer-Verlag, New York, Heidelberg, 1972. Translated from the second German edition by William H. Ruckle. 14 